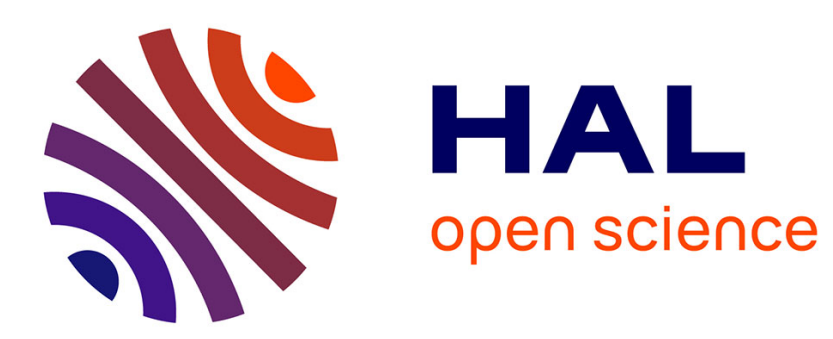

\title{
Fictions du capitalisme dans le récit d'entreprise contemporain
}

\author{
Corinne Grenouillet
}

\section{To cite this version:}

Corinne Grenouillet. Fictions du capitalisme dans le récit d'entreprise contemporain. Les Fables du politique des Lumières à nos jours, Presses Universitaires de Strasbourg, pp.191-203, 2012. hal03151707

\section{HAL Id: hal-03151707 https://hal.science/hal-03151707}

Submitted on 25 Feb 2021

HAL is a multi-disciplinary open access archive for the deposit and dissemination of scientific research documents, whether they are published or not. The documents may come from teaching and research institutions in France or abroad, or from public or private research centers.
L'archive ouverte pluridisciplinaire HAL, est destinée au dépôt et à la diffusion de documents scientifiques de niveau recherche, publiés ou non, émanant des établissements d'enseignement et de recherche français ou étrangers, des laboratoires publics ou privés. 


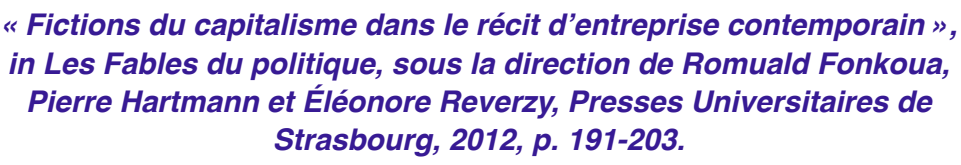

\title{
Fictions du capitalisme dans le récit d'entreprise contemporain
}

\author{
Corinne Grenouillet*
}

L appellation "récits d'entreprise» désignera ici des textes en prose, de longueur variable, à dominante fictionnelle, qui placent leur diégèse dans le cadre du monde du travail (l'usine, le chantier, le garage, le bureau, l'agence...). Ces récits n'adhèrent que rarement à l'esthétique réaliste ou naturaliste, mais relèvent du réalisme comme posture: tous, à divers titres, envisagent la littérature comme moyen de connaissance du monde ${ }^{1}$.

Le travail, qui renvoie à des lieux, des activités humaines et un temps spécifiques, concentre a priori les différents aspects du politique, au sens large. On sait que les relations de pouvoir, de subordination, d'exploitation et de domination dans le cadre de l'entreprise réelle rejouent celles qui régissent la vie des hommes hors de ce secteur: par exemple la manière de gérer le pouvoir dans les entreprises jusque dans les années 1970-1980 (rigidité de la hiérarchie, de la planification, lourdeur bureaucratique et refus de prendre en compte les avis et les singularités individuels) diffracte le paradigme politique dominant (l'État technocratique).

Les transformations du monde du travail, voire sa «déconstruction» - par le biais de la précarisation de l'emploi, de la "dualisation du salariat» (d'un côté les salariés pourvus d'un CDI, de l'autre ceux qui courent de stages ou de "missions" d'intérim, en CDD) ou de l'intensification des rythmes de travail - manifestent

Université de Strasbourg.

1 Nous ne prendrons donc pas en compte le sens commun et récent de «récits d'entreprise» ou "biographies d'entreprise», termes qui désignent des textes réalisés pour le compte d'entreprises réelles: ils prennent leurs locaux réels pour décor, leurs personnels pour personnages, leurs activités pour support de fiction, dans un but publicitaire ou managérial. 
avec intensité les mutations de la démocratie et du système capitaliste dans lesquels nous vivons ${ }^{2}$.

Depuis une vingtaine d'années, les «récits d'entreprise» convoquent les procédés traditionnels de la fiction, ne rechignent pas devant la constitution de personnages, ni l'instauration d'une fable ${ }^{3}$. Celle-ci est souvent réduite à une anecdote, un mince fil narratif exploité dans la nouvelle, le texte court ou fragmenté: Chantier de Gisèle Fournier, 2006; Yves Pagès, Petites natures mortes au travail; Christophe Fourvel, Des hommes, 2003; Hélèna Villovitch, Dans la vraie vie (2005) ou L'Entreprise, sous la direction d'Arnaud Viviant (2003). L'anecdote peut y être pulvérisée dans une démarche "post nouveau roman" de mise en cause du récit (Thierry Beinstingel, Composants, 2002) ou celui-ci peut viser au contraire à une essentialisation et une épure (François Emmanuel, La Question humaine, 2000). Une autre catégorie est celle des textes romanesques (par leur longueur), lesquels peuvent d'ailleurs témoigner d'une expérience personnelle largement transposée au plan de la fable, tels François Bon (Sortie d'usine, 1982), Michel Houellebecq (Extension du domaine de la lutte, 1994), Jean-Pierre Martin (Le Laminoir, 1995), François Salvaing (La Boîte, 1998), Frédéric Beigbeder (99francs, 2000), Christophe Fourvel (Des Hommes, 2003), Dominique Manotti (Lorraine connection, 2006), Lydie Salvayre (La Médaille, 1993) etc.

Soucieuse de dégager une tendance de la littérature contemporaine, nous émettons l'hypothèse que ces récits, centrés sur le monde du travail ou plaçant des personnages dans son orbe, allégorisent plutôt les mutations ouvertes par le «troisième esprit du capitalisme». Or capitalisme et libéralisme ne sont pas des systèmes politiques, mais économiques, et touchent moins la politique que le politique. La question politique n'est d'ailleurs pas ou rarement posée frontalement dans notre corpus. Les trois niveaux que nous examinerons (les structures formelles et énonciatives, l'espace représenté, enfin la fable elle-même) concernent moins un fonctionnement allégorique stricto sensu que la constitution de la fiction. Nous verrons par quels moyens proprement littéraires ces récits décrivent la douloureuse situation de l'individu au travail dans un monde régi par le capitalisme et la démocratie libérale.

2 L. Boltanski et È. Chiapello, Le Nouvel esprit du capitalisme, Gallimard, coll. "nrf essais», 1999 (sur la déconstruction du monde du travail, voir p. 291 et sq.). Sur la définition de la "condition" qui est la nôtre, voir M. Gauchet, La Condition historique, Entretiens avec François Azouvi et Sylvain Piron, Stock, coll. "Les Essais», 2003, 355 p.

3 Sont donc volontairement délaissés les textes fondés sur des enquêtes auprès de travailleurs réels dont les témoignages constituent ensuite la matière première de textes hybrides, parfois dénommés romans par leurs auteurs tels Daewoo de F. Bon (Fayard, 2004). 
Une grande partie de ces romans présente la particularité énonciative d'être à la première personne: l'effet personnage et ses implications sur le code de sympathie du lecteur ${ }^{4}$ en sont évidemment renforcés, option largement partagée par la littérature contemporaine.

Dans Extension du domaine de la lutte, un salarié d'une grande entreprise informatique guetté par l'épuisement vital analyse les relations humaines et professionnelles d'un point de vue distancié, voire désabusé, souvent satirique: il associe le lecteur à son point de vue d'observateur toujours en retrait d'un monde jugé et apprécié. Cette posture d'extériorité du personnage par rapport au monde du travail dans lequel il évolue se retrouve dans La Question humaine récit dans lequel elle converge avec la mise en place d'un statut d'enquêteur. Chargé de mener une enquête sur la santé mentale du directeur de son entreprise, le narrateur, un psychologue rattaché aux ressources humaines, s'aperçoit que Karl Jüst est littéralement obsédé par le lourd passé nazi de la génération qui l'a précédé. L'enquête biographique emprunte alors le registre du roman policier: le narrateur rencontre successivement des personnages proches du directeur et le directeur lui-même afin de percer le mystère de sa personnalité. Ces récits jouent sur l'assimilation potentielle entre narrateur et auteur, renforcée par la connaissance que nous avons des expériences ou statuts professionnels de ces écrivains (François Emmanuel est psychothérapeute, Houellebecq a travaillé chez Unilog et au ministère de l'agriculture, François Bon a une expérience industrielle après une formation aux Arts et métiers d'Angers).

Certains des romans de notre corpus adoptent des dispositifs énonciatifs plus sophistiqués ou plus originaux: Des Hommes, écrit sur le mode du tu (comme La Modification), construit une incertitude sur le statut du narrateurfrère du "personnage" ou narrateur abstrait? Dans Le Laminoir, le narrateur qui a pris la parole pour raconter l'histoire de Simon, établi en usine dans les années 1970, devient peu à peu un personnage à part entière qui tend à prendre la première place. Composants (2002) se situe dans la lignée impersonnelle du Robbe-Grillet de La Jalousie: la présence d'une subjectivité est pourtant constante alors même que le récit ne comporte aucune marque d'énonciation, privilégiant l'impersonnalité du on et l'utilisation de structures stylistiques récurrentes telles les phrases a-verbales ou les énumérations. Le lecteur découvre ainsi, par les yeux d'un intérimaire de banlieue issu de l'immigration maghrébine, une semaine de travail ordinaire l'obligeant à passer deux nuits à l'hôtel loin de sa famille. Dans La Médaille, huit "allocutions" prononcées par des représentants de la structure d'encadrement d'une entreprise de construction automobile au cours d'une cérémonie de remise de médailles du travail alternent avec sept «réponses» de médaillés dans un dispositif continuellement ironique: 
la réponse du médaillé vient contredire, de façon systématique, les discours des managers censés galvaniser l'énergie des travailleurs et les compliments adressés par la direction.

Le point commun de ces dispositifs est d'inviter à considérer l'entreprise d'un point de vue subjectif, au travers duquel l'écrivain interroge la réalité des rapports socio-économiques productrice de souffrance psychologique sinon physique (dépression, solitude, alcoolisme, mais aussi maladie grave); ils tendent aussi à donner la vision, parcellaire, de celui qui travaille, isolé, simple composant d'un monde sur lequel il n'a plus, aujourd'hui, de prise... particule élémentaire individualisée et solitaire à l'image de l'homme démocratique de nos sociétés modernes ${ }^{5}$.

Ces choix revêtent une dimension politique dans le sens où ils font entendre la voix des dominés, leurs points de vue, leurs perceptions, leurs analyses. L'intérimaire de Beinstingel, l'ouvrier de Bon, l'employé des chemins de fer de Fournel, le cadre moyen chez Houellebecq sont autant de nouvelles corporations subjectivées par le roman d'entreprise. Les voix qui surgissent de l'usine (chez François Bon), qui s'élèvent, grotesques ou pathétiques, parmi les médaillés du travail de Lydie Salvayre, posent une revendication littéraire et politique: l'égalité démocratique. C'est peut-être chez cet auteur que l'affrontement des voix revêt le plus franchement une dimension politique, au sens conflictuel que Rancière accorde à ce terme ${ }^{6}$ : chaque «réponse» conteste le rôle que l'allocution officielle vient d'attribuer au récipiendaire en révélant «l'envers de la médaille», la souffrance et la violence, la solitude et la détresse.

L'espace de ces récits est l'objet d'un important investissement symbolique, qu'il s'agisse de l'usine close et mortifère décrite par François Bon, coupée de l'espace domestique et privé (et dont il faut à tout prix sortir comme l'indique le titre du roman), ou des lieux marqués par la porosité instaurée entre espace

5 Voir L. Steiner, Sade-Houellebecq, Du boudoir au sex-shop, L'Harmattan, coll. "Approches littéraires", 2009.

6 Dans La Mésentente, Galilée, 1995, J. Rancière insiste sur le nécessaire conflit existant sur le «partage du sensible» qui est, selon lui, au cœur même de la définition du politique. Nos analyses rejoignent celles d'A. Chauvin, "Voix ouvrières dans Mémoires de l'enclave (Jean-Paul Goux) et Daewoo (François Bon)", Les Voix du peuple dans la littérature des XIX et XXe siècles, C. Grenouillet et É. Reverzy dir., PUS, 2006, p. 361-377, J.-P.Engelibert «Ressources inhumaines: le nouvel esprit du travail dans quatre romans français contemporains», Trans $n^{\circ} 4$ : À quoi bon la littérature?, 2007 (http://trans.univ-paris3.fr/) et R. Michel, "Le Bonheur des mots pour dire le malheur des corps: F. Bon, Daewoo", Recherches textuelles $n^{\circ}$ 7: Les Voix du peuple et leurs fictions, A. Petitjean et J.-M. Privat éd., 2007, p. 107-137. 
professionnel et espace privé (Villovitch, Viviant, Houellebecq, Beigbeder). L'espace du travail fictif interroge la place assignée à l'individu dans la société et dessine les contours d'une nouvelle ère du capitalisme.

De la boîte ou la taule dont on ne sort que mort ou gravement accidenté (Bon, Salvaing), on passe aux bureaux d'où l'on est renvoyés, et aux extérieurs d'immeubles au pied desquels la narratrice mange un sandwich car, en contrat à durée déterminée, elle n'a pas le droit de pénétrer dans la cantine réservée aux salariés embauchés en CDI (Villovitch). Des lieux paradoxaux (gare où les trains ne s'arrêtent plus dans Des hommes; chaîne de montage en cours de déménagement dans Daewoo) peuvent manifester l'impossibilité pour l'individu de s'inscrire dans les nouveaux territoires du capitalisme que sont les "réseaux ${ }^{7}$ ".

Le narrateur d'Extension du domaine de la lutte travaille dans une entreprise informatique, emblématique du nouvel esprit du capitalisme; la dimension immatérielle du produit vendu, l'importance accordée aux rencontres et aux réunions de travail, ainsi qu'à l'information (objet d'une théorie de la part d'un personnage secondaire) renforcent l'apparentement du narrateur (et de son collègue) à l'homme léger, l'homme des réseaux décrit par Boltanski. Il est d'ailleurs présenté à deux reprises comme "l'homme système $e^{8}$. Mobile et sans attache affective (il a rompu avec sa première femme, n'a pas conservé de liens avec sa famille), il est conduit à de nombreux déplacements professionnels en Province et de multiples rencontres. Son collègue de travail se nomme Tisserand: le tissage contenu dans son patronyme suggère l'idée de réseau et suffit à faire du personnage un gagnant sur le plan économique (comme le dit explicitement le narrateur, et comme son habileté lors de réunions le confirment $\left.{ }^{9}\right)$. Légèreté, rapidité, capacité à établir des connexions nouvelles (voir la mobilité de Jean-Yves Fréhault, le "penseur de l'informatique», qui quitte sa boîte pour une autre), telles sont les qualités des cadres décrits par Houellebecq parfois efficacement renforcées par la beauté physique, comme celle, subjuguante, d'un chef de service ${ }^{10}$. Ce monde de gagnants toujours en mouvement est évidemment croqué sur le mode satirique - tel ce «chef de service» qui semble glisser et non marcher, la "cravate penchée de côté, comme pliée par le vent de la course ${ }^{11}$ » - et du point de vue d'un narrateur sombrant, à l'inverse, dans la dépression.

Relatant le parcours professionnel d'un responsable des Ressources humaines dans une grande société spécialisée en emballage, François Salvaing met également en scène dans La Boîte la multiplicité de lieux où se déroule une carrière

\footnotetext{
7 L. Boltanski et È. Chiapello, op. cit., p. 111 et suivantes; p. 177 et sq.

8 M. Houellebecq, Extension du domaine de la lutte, Éd. Maurice Nadeau, coll. "J'ai lu", 1994, p. 53

$9 \quad$ Ibid., p. 56 et p. 60.

10 Ibid., p. 24 et p. 39.

11 Ibid., p. 36
} 
de "gagnant». Ce personnage est mobile à tous les sens du terme: il se déplace sur le territoire français et progresse dans la hiérarchie de son entreprise; sa femme, cadre supérieure elle aussi, refuse la maternité et gravit les échelons hiérarchiques de conserve avec son mari.

Cette mobilité inférée par la "Nouvelle Donne ${ }^{12}$ » dessine une ligne de partage entre la légèreté aérienne de ceux qui se déplacent de lieux en lieux - voir qui disparaissent ${ }^{13}$, et ceux qui sont attachés à une tôle, voire à une machine; la dimension obsidionale de l'usine, "tôle d'un peu plus de mille bonhommes ${ }^{14}$ ", dont la «poterne» d'entrée est surveillée par des «matons» ou un gardien "arme au flanc ${ }^{15}$ " est un motif récurrent de Sortie d'usine (écrit une dizaine d'années plus tôt que les deux romans précédents): cet "enfermement ici de l'homme avec d'autres hommes ${ }^{16}$ » induit des comportements d'aliénés, intellectuellement et affectivement "emmurés ${ }^{17}$ ". L'usine est dépeinte comme un lieu mortifère; les événements saillants de ce roman essentiellement descriptif sont les morts d'ouvriers, remorqués sur des transpalettes au cours d'une cérémonie qui réactive l'intertexte mythique: le Passage. Qu'il s'agisse de la mort du sourd venu «crever à son premier repas des anciens [...] à l'usine ${ }^{18}$ » $-\mathrm{du}_{\text {caissier }}{ }^{19}$ ou du vieux patron ${ }^{20}$, l'usine se transforme en "ventre à bruit", les ouvriers tapant de leurs outils, sifflant et utilisant l'air comprimé pour faire le plus de vacarme possible. La mort d'un délégué syndical, est traitée, elle, sur un autre mode: le pastiche du discours nécrologique. La "quatrième [et dernière] semaine» voit l'opposition violente entre un ouvrier déprimé et un contremaître se régler à coups de marteau; le suicide de la victime (l'ouvrier) est suggérée ${ }^{21}$ avant que le récit, soudain à la première personne, ne mette le narrateur en face de son propre tombeau.

Contrairement à la démultiplication des lieux, l'usine de Bon assigne une place fixe à l'être (derrière sa machine: «le visage s'était associé à son ordinaire, une place, atelier machine, couleurs geste $\left.{ }^{22} »\right)$; l'ouvrier est constamment sous surveillance jusque dans les toilettes dont on a coupé le bas des portes. La grève

12 F. Salvaing, La Boîte, Fayard, 1998, p. 150.

13 Voir le titre du roman de F. Vigouroux, Monsieur le Président, pourquoi nous as-tu abandonnés? (PUF, 2003) qui est une question adressée à un dirigeant d'entreprise disparu.

14 F. Bon, Sortie d'usine, Éd. de Minuit, 1982, p. 45.

15 Ibid., p. 165.

16 Ibid., p. 52.

17 "emmurés de l'interdit posé sur le toucher, sauf la main», ibid., p. 52.

18 Ibid., p. 79.

19 Ibid., p. 90.

20 Ibid., p. 98.

21 Ibid., p. 154.

22 Ibid., p. 117. 
permet la libération du mouvement, mais toujours à l'intérieur de l'usine: les grévistes pénètrent alors dans tous les endroits généralement interdits. Comme ses compagnons, le narrateur fomente le projet de "s'en sortir ${ }^{23}$ ", de "se sauver de l'enfermement de cette réalité close ${ }^{24}$ ", accompli dans le dernier chapitre: la libération ne passe donc pas par l'action militante (la grève) puisque celle-ci ne permet que d' "occuper ${ }^{25}$ » différemment le même lieu, de l'intérieur duquel on s'est "barricadé ${ }^{26}$ ». "L'usine comme une évidence sur elle-même enclose ${ }^{27}$ " n'a de sens que pour celui qui y travaille. Sortie d'usine offre la vision d'un lieu aliénant, de l'enfermement ${ }^{28}$ et de la contrainte. Douze ans plus tard, dans Daewoo, les usines sont devenus des "usines tournevis", facilement démontables et qui semblent avoir été "posées là par hélicoptère ${ }^{29}$ »: la "ligne de fabrication" de l'usine de Daewoo-Fameck est en partance pour "la Turquie ${ }^{30}$ » puis une grue retire progressivement les lettres formant le mot DAEWOO; l'usine et ses symboles s'envolent, au sens propre et au sens figuré, symboles qui rejoignent les impératifs de mobilité et de légèreté prônés par le nouvel esprit du capitalisme dont François Bon montre le revers en interviewant des ouvrières licenciés.

Composants s'intéresse - cas rare ${ }^{31}$ - au travail de l'intérimaire, travailleur tournevis (s'il en est) déplaçable de lieu en lieu, sans avoir aucune emprise sur cette mobilité subie. Le récit place son personnage (jamais nommé) pendant une semaine au cœur du territoire déshumanisé d'un bord de ville. Car le travail est désormais "rassemblé» dans "des pôles d'activités, des espaces de création, des pépinières d'entreprise, tout un vocabulaire de parc d'attraction». Et le nar-

23 Ibid., p. 127.

24 Ibid., p. 165.

25 Ibid., p. 116.

26 Ibid., p. 110.

27 Ibid., p. 162.

28 Ce motif obsidional n'est pas en soi une nouveauté. Un écrivain prolétaire, Gauny, menuisier marqueteur de son état, dans "Le travail à la tâche» (années 1840) établissait déjà un parallèle entre prison et manufacture. En 1830, la prison cellulaire constituait une nouveauté, caractérisée par l'impossibilité d'une parole (les prisonniers ne peuvent échanger, les sons se perdent), par un dispositif de surveillance panoptique et par l'éclairage des cellules la nuit, qui empêche l'obscurité permettant la méditation. Ces nouvelles prisons d'où on ne peut s'évader sont envisagées comme des sépulcres. (Voir l'analyse de J. Rancière, La Nuit des prolétaires. Archives du rêve ouvrier, Fayard, 1981, p. 97 et sq.).

29 F. Bon, Daewoo, Fayard, 2004, p. 73.

30 Ibid., p. 79.

31 Il existe peu de récits littéraires traitant de la situation de l'intérimaire, hormis Temps machine de F. Bon, Verdier, 1993 et les nouvelles d'Y. Pagès (Petites natures mortes au travail, Verticales / Le Seuil, 2000) ou d'Hélèna Villovitch (Dans la vraie vie, Éd. de l'Olivier, 2004). On notera aussi le témoignage, de grande qualité d'écriture, de Daniel Martinez, Carnets d'un intérimaire (préface de Michel Pialoux, Marseille, Agone, coll. "Mémoires sociales", 2003). 
rateur de commenter: "Et cette impression que le boulot est devenu une chose légère et futile, aussi intéressante que se promener à Disneyland ${ }^{32}$ ". L'intérimaire se retrouve seul, isolé toute la journée sur son lieu de travail, entrepôt où il doit ranger des boîtes. C'est une autre forme de déshumanisation qui est saisie dans ce texte, celle liée à la répétition monotone des mêmes gestes durant une semaine: des listes, des énumérations d'objets (les composants à ranger), la description méticuleuse des décors et des matières aboutissent à une fusion de l'intérimaire avec son cadre de travail, comme l'indiquent ses "mains de carton" ou la façon dont un syndicaliste venu vérifier la conformité de l'installation avec des normes de protection des ouvriers «salue à la cantonade l'intérimaire et les étagères ${ }^{33}$ ".

Hormis des repas pris fortuitement avec d'autres travailleurs «à la gamelle ${ }^{34}$ », parfois "sans paroles», l'intérimaire est coupé de toute forme de fraternité humaine. Cette solitude, également celle du narrateur de Houellebecq considérant sa boîte de Saupiquet ${ }^{35}$, est le thème central du documentaire Notre pain quotidien, consacré à l'industrie agro-alimentaire (Nikolaus Geyrhalter, 2005), où l'on voit en leitmotiv des travailleurs manger seuls face à la caméra. Dans Composants, cet isolement est renforcé par le monde objectal décrit et par l'enfermement dans un décor marqué au sceau de la verticalité (les tas d'objets à ranger sont comme des "gratte-ciel de Manhattan" qu'il faut réduire en maisons $\left.{ }^{36}\right)$. Il devient complet lors d'une soirée et une nuit passées dans des «nonlieux»: cafétéria de supermarché et hôtel à bas prix («Fasthôtel»). L’entreprise n'existe plus ici comme un endroit - toujours le même - où l'on travaille et où différentes formes de fraternité aident à supporter le "malheur" de la condition ouvrière $^{37}$, mais comme structure vague et impalpable où la précarisation renforce la solitude accablante de l'être.

Se situer paradoxalement à l'extérieur et à l'intérieur d'une entreprise caractérise le CDD décrit avec beaucoup d'humour par Hélèna Villovitch. Sa nouvelle "Qu'est-ce que tu vas faire?» montre la difficulté d'intégration d'une jeune femme dans les entreprises qui l'accueillent pour des contrats très courts (elle n'a pas d'ordinateur, pas de chaise de bureau etc.). Elle met en évidence la porosité entre l'espace professionnel et l'espace intime en construisant astucieusement un parallélisme entre ces deux espaces. Les quatre chapitres, sont de plus en plus courts, à mesure que les CDD de la narratrice se raccourcissent également (passant de trois mois, à un mois, puis trois jours et enfin quatre heures). Ses amours

32 T. Beinstingel, Composants, roman, Fayard, 2002, p. 9.

33 Ibid., p. 73.

34 Ibid., p. 31.

35 M. Houellebecq, Extension du domaine de la lutte, op. cit., p. 27.

36 T. Beinstingel, Composants, roman, op. cit., p. 115. Voir aussi p. 61 et 103.

37 Voir les analyses de S. Weil, La Condition ouvrière, 1951. 
sont, elles aussi, de plus en plus précaires; la narratrice connaît une conjugalité de plus en plus sommaire: après les «six jolies assiettes en porcelaine ${ }^{38}$ " de Jacques, elle passe "aux assiettes en carton" avec Karim: "Ensuite les assiettes passent directement à la poubelle et moi à la casserole ${ }^{39}$ ». Avec Fred, successeur de Karim, elle établit une relation basée sur le plaisir immédiat, décrite avec un vocabulaire propre au travail: "Au bout du compte, la mission est accomplie avec efficacité, mais sans excès de zèle ${ }^{40}$ ». Enfin, dans le dernier chapitre la narratrice remarque un jeune type en face d'elle dans un train. Aux toilettes, «Nous concluons rapidement $[\ldots]^{41}$ ». Cette homologie structurelle rend palpable sur un mode fictionnel ludique l'établissement d'une corrélation entre la durée des engagements contractés dans la vie sentimentale et dans la vie professionnelle observée par les sociologues ${ }^{42}$.

On peut s'étonner que face à un tel sujet (l'homme au travail), qui hérite d'un investissement politique majeur depuis plus d'un siècle, les récits contemporains répugnent à une approche politique "directe", qui emprunterait par exemple le mode discursif (le narrateur ou les personnages prenant la parole pour énoncer des théories).

Les romans de notre corpus évitent avec une systématicité notable les anciennes catégories d'analyse, en particulier issues du marxisme, même s'ils peuvent prêter à tel ou tel personnage une expérience de l'engagement militant. Les thèmes traditionnellement "politiques" comme la grève ou la manifestation, susceptibles de dynamiser des intrigues souvent minces, sont présents mais toujours secondaires, comme si ces auteurs refusaient d'aborder la condition ouvrière sur un mode politique jugé daté ou au moyen de motifs littéraires périmés. Fil directeur de la "troisième semaine» de Sortie d'usine, la grève ne donne aucune tension narrative au roman: elle est l'occasion de dépeindre un lieu occupé autrement, mais tout aussi obsidional.

38 H. Villovitch, Dans la vraie vie, op. cit., p. 27.

39 Ibid., p. 29.

40 Ibid., p. 33. Je souligne.

41 Ibid., p. 34.

42 "Le retard à l'entrée dans la vie professionnelle et la substitution des embauches au coup par coup aux modes d'insertion ouvrant la perspective d'une carrière, sont allés de pair avec un développement des engagements à court terme dans la vie privée comme le montrent non seulement la diminution du nombre des mariages et l'augmentation de celui des divorces mais aussi la fragilité croissante des liaisons instaurées "sans papier" et définies par la "cohabitation" " (L. Boltanski et È. Chiapello, op. cit., p. 506) 
Si la grève est devenue irracontable parce que renvoyant à une tradition du roman social jugée aujourd'hui dépassée ${ }^{43}$, il semble que ce soit l'ensemble des motifs directement politiques qui tendent à s'estomper, ou alors à être traités sur le mode de la distance ironique. Dans La Médaille par exemple, les ouvriers qui se sont progressivement révoltés en arrière-fond de la remise des médailles du travail, pénètrent dans la salle et exigent des médailles... en chocolat. L'émeute est enfin annihilée par l'habileté d'un ingénieur qui promet «l'embauche à vie» aux ouvriers "les plus méritants", fait valoir la "collaboration» et le caractère «totalement démodé» de la révolte ${ }^{44}$. La fin du roman correspond aux "applaudissements enthousiastes de tous ", c'est-à-dire émeutiers, direction et médaillés. L'engagement politique est raillé sans méchanceté dans Le Laminoir: une allégorie s'y affiche comme telle sur un mode ludique mettant à distance les anciens schémas d'interprétation politique. Simon, jeune étudiant, que nous découvrons dans les premières pages dans un amphithéâtre parisien écoutant Jankélévitch (célèbre entre autres pour son livre... sur l'ironie), se voit subjugué par l'apparition de Mado, allégorie de la classe ouvrière on ne peut plus sexy:

[...] Des boucles en forme de clé à molettes ornent des oreilles admirables; des bracelets d'acier trempé décorent des poignets aristocratiques [...] une taille fait au tour met en valeur des seins de fraise, et le visage par-dessus est parfaitement ajusté ; le tout, moulé dans un bleu de chauffe $[. . .]^{45}$

Elle s'adresse à Simon qui se sentira bientôt «envoûté ${ }^{46}$ »:

[...] Entends le chant des métallos. Il te faut changer de peau, petit couillon. Casse-toi. Prends des risques. Rends-toi utile. Mets la main à la pâte. Tu me cherches depuis longtemps. Je suis la Classe ouvrière ${ }^{47}$.

Cette allégorie est d'autant plus significative qu'elle intervient dans un contexte (le livre est publié en 1995) où la notion même de classe ouvrière, qui suppose une analyse marxiste de l'exploitation d'une classe par une autre, tend à disparaître de tous les discours critiques. Elle a pour fonction de dater l'engagement de Simon; elle désigne son sentimentalisme, ses illusions, la valeur de mythe de ses anciennes croyances comme s'il était aujourd'hui impossible de parler de la classe ouvrière autrement qu'avec un sourire (signifiant une prise de distance d'avec le caractère jugé éculé de cette notion) ou en termes de disparition ou de conservation de la mémoire d'un âge révolu. Un autre établi est d'ailleurs cible

43 Pourtant reprise et revivifiée par G. Mordillat dans Les Vivants et les morts (Calmann-Lévy, 2004). Voir aussi Le Roman social: Littérature, histoire et mouvement ouvrier, sous la direction de S. Béroud et de T. Régin, Les Éd. de l'Atelier, 2002.

44 L. Salvayre, La Médaille, Le Seuil, 1993, p. 157 et p. 161.

45 J.-P. Martin, Le Laminoir, Seyssel, Champ Vallon, 1995, p. 22-23.

46 Ibid., p. 25.

47 Ibid., p. 23. 
d'ironie dans notre corpus: un ingénieur de l'entreprise Bisson (dans La Médaille) dont la ferveur révolutionnaire d'antan s'est muée en la conviction que seule la collaboration individuelle à l'effort de l'entreprise valait d'être menée. La trajectoire de ce personnage qui a «réussi» révèle combien le capitalisme contemporain a su récupérer et intégrer pour son profit les éléments de la critique sociale comme de la critique «artiste» des années 1960-197048.

Deux romans du corpus privilégient l'énoncé d'idées ou d'analyses à teneur politique: Extension du domaine de la lutte et La Question humaine. Houellebecq à qui les «réflexions théoriques [...] apparaissent comme un matériau romanesque aussi bon qu'un autre, et meilleur que beaucoup d'autres ${ }^{49}$ " ne manque pas de prêter à la plupart de ses personnages, à commencer par son narrateur, des théories plus ou moins élaborées, énoncés sentencieux, présentés avec une dose de sérieux variable et dont certaines puisent dans la tradition philosophique de l'apologue schopenhauerien. Comme plus tard Les Particules élémentaires, son récit recèle plusieurs saynètes allégoriques dont l'interprétation est souvent délicate, car elles relèvent de dispositifs énonciatifs hétérogènes qui empêchent l'adhésion du lecteur ${ }^{50}$. Parmi les théories présentant un certain gage de sérieux et que corroborent plusieurs épisodes du roman, celle qui donne son titre au livre utilise la métaphore spatiale pour montrer que tout le malheur de l'homme contemporain vient du fait que le libéralisme s'est étendu du domaine économique au domaine sexuel, provoquant des inégalités scandaleuses entre gagnants et perdants, les gagnants sur le plan économique (tel Tisserand) pouvant d'ailleurs parfaitement être des perdants sur le plan sexuel ${ }^{51}$. Cette métaphore est soutenue par une courte allégorie, également d'ordre spatial, et qui tire le roman du côté philosophique davantage que politique: entrer dans le domaine de la lutte est comme tenter de traverser une rivière d'eau froide; le nageur arrivé loin du bord, l'existence d'une autre rive ne lui apparaît plus comme certaine. "Vous continuez à nager pourtant, et chaque mouvement que vous faites vous rapproche de la noyade ${ }^{52}$ ». Sur fond de philosophie pessimiste, voire nihiliste, Houellebecq met en fiction le politique contemporain: individualisation et solitude, augmentation de la concurrence interindividuelle, marchandisation de l'espace privé, standardisation de l'être humain sous l'effet de l'entrée dans une nouvelle ère économique...

48 Selon les termes utilisés par L. Boltanski et È. Chiapello, op. cit.

49 M. Houellebecq, Interventions, Flammarion, 1998, p. 7.

50 Je me réfère aux «fictions animalières» dans lesquels des animaux, chimpanzé ou caniche, énoncent des théories en s'exprimant dans un style boursouflé à effet comique qui jette d'emblée le discrédit sur les théories énoncées.

51 M. Houellebecq, Extension du domaine de la lutte, op. cit., p. 100.

52 Ibid., p. 14. 
La Question humaine entend utiliser l'histoire du nazisme pour donner une clé d'interprétation du fonctionnement de l'entreprise moderne. Cette fois-ci, c'est la construction de la fable qui suscite l'interprétation, non les discours du narrateur-, le récit d'Emmanuel conduit le lecteur à admettre l'idée d'un continuum entre la gestion des entreprises d'aujourd'hui et celle de la solution finale mise au point par les nazis autrefois. Continuum de la logique mise en œuvre tout d'abord: les procédés sont les mêmes, sélection et mise à l'écart des plus faibles, et surtout utilisation d'une langue qui dénie la cruauté des actes commis et masque les conséquences de ceux-ci à leurs exécutants. Continuum historique enfin: les deux directeurs de l'entreprise, ainsi qu'un cadre licencié autrefois, allemands tous trois, assument mal l'héritage historique de la génération précédente. Pourtant, le récit se montre totalement indécidable sur plusieurs points décisifs, ce qui montre une résistance du texte à une lecture trop univoque. En effet, le lecteur ne peut trancher par exemple si Mathias Jüst - personnage fictif - est bien le fils du bureaucrate réel qui suggéra des améliorations aux camions à gaz ou un simple homonyme; si Karl Rose, dont le véritable patronyme demeure un mystère, est ou non un enfant Lebensborn; si la filiation d'Arie Neumann, récemment licencié, l'inscrit du côté des victimes juives ou au contraire du côté des bourreaux nazis.

La Question humaine relate le parcours d'un psychologue industriel adhérant totalement aux valeurs du capitalisme mondialisé et amené à rompre avec elles (donc à quitter son travail) pour se consacrer au soin d'enfants autistes: cette trajectoire, exemplaire, rattache le récit à la structure d'apprentissage du roman à thèse. Le recours à l'Histoire, en particulier à la citation du document historique, s'inscrit dans une structure narrative fondée sur le dévoilement (pour le narrateur-enquêteur, pour le lecteur). Celui-ci concerne pour l'essentiel la langue utilisée par les manuels de psychologie du travail, et que le narrateur a faite sienne. Cette langue, qui est celle du management, fonctionne sur le principe de l'euphémisation, voire de la dissimulation de la réalité. Elle utilise «des mots propres qui ne tachent pas ${ }^{53}$ ": le "chargement" (Ladung) mentionné dans la note du 5 juin 1942 évite de nommer les êtres humains chargés dans le camion à gaz; de même le mot "restructuration" n'intègre pas les licenciements qui en sont le corollaire inévitable.

La conclusion à laquelle le lecteur est conduit est la suivante: le vocabulaire technique utilisé par le management moderne parvient à créer une zone de "déresponsabilisation" qui permet aux principaux acteurs d'une restructuration industrielle - comme autrefois aux officiers SS - de ne pas se sentir concernés par les conséquences de leurs actes, pas plus sur le plan humain (la fameuse «question humaine» du titre), que sur le plan moral ou juridique. Il ne s'agit 
donc pas exactement de "comparer» les cadres des entreprises modernes aux "officiers nazis» (ce dont Emmanuel fut vertement accusé par toute une partie de la critique), mais de montrer plus subtilement la permanence d'un dispositif linguistique et psychologique propre à notre modernité bureaucratique et industrielle ${ }^{54}$. Il nous semble injuste de réduire ce livre épuré et remarquablement construit à la "lourdeur" de la thèse qui y serait exprimée.

À la fin du roman, quitter son travail revient pour le narrateur à poser un acte de «résistance intime à Tiergarten 4 ", autre texte nazi cité ${ }^{55}$ : Simon choisit donc son camp: les "marges du monde». Le terme de "résistance» connote qu'il s'agit là d'un engagement politique; Simon a redéfini sa place et son rôle au sein de la cité.

Le récit d'entreprise se saisit des nouveaux types sociaux produits par notre système économique et politique, auxquels elle donne "démocratiquement» la parole, qu'ils figurent parmi les gagnants (homme système, golden boy, cadre directeur ou moyen) ou parmi lesperdants de la "Nouvelle donne» (CDD, ouvrier, intérimaire)...

En utilisant l'espace du travail comme cadre de la fiction, ils s'attachent à interroger la place et le rôle de l'homme dans nos sociétés démocratiques. Le lieu (littéraire) du travail semble ainsi un espace privilégié de la mise au jour du système ou de la logique qui sous-tend l'organisation fondamentale de nos sociétés contemporaines. Cette mise au jour, à visée critique, tente de frayer des voies fictionnelles nouvelles par le biais de la satire, de l'ironie ou le renouvellement des formes narratives.

L'entreprise fictive symbolise un microcosme où se joue à taille réduite la solitude de l'être démocratique, jeté dans le monde de la concurrence inter-individuelle, réifié par le système économique et politique, dépossédé des cadres anciens et structurants de la vie collective.

54 Sur ce point, la thèse développée sur le mode fictif par F. Emmanuel rejoint les analyses de Z. Bauman dans Modernité et holocauste (La Fabrique, 2002) ou de C. Dejours dans Souffrance en France: La Banalisation de l'injustice sociale (Le Seuil, coll. "L'Histoire immédiate», 1998). Tous deux s'appuient sur les travaux de Hannah Arendt et son analyse du cas Eichmann.

55 Le programme Aktion Tiergarten 4, mis en place dès 1939, procédait à l'élimination de "malades incurables" allemands, la plupart malades mentaux. 